\title{
DIE KOMMUNALE SELBSTVERWALTUNGSREFORM IN BERLIN
}

\author{
Yrd. Doç. Dr. Ayşegül KAPLAN*
}

\section{EINFÜHRUNG}

Berlin ist die neue Hauptstadt der vereinigten Deutschland und auch eine wichtige Metropole von Europa. Als eines von sechzehn Ländern hat Berlin eine Landesregienung, sie heisst Senat von Berlin. Unter dem Senat arbeitet die Hauptverwaltung, vergleichbar den Ministerien in den anderen Ländem. Der Regierende Bürgermeister ist der Leiter von der fünfzehn Senatsverwaltungen ${ }^{1}$ (wie Senatsverwaltung für Finanzen, für Inneres, für Justiz, für Soziales, für Gesundheit, für Bau- und Wohnunswesen, für Jugend und Familie etc.) Der Senat nimmt durch die Hauptverwaltung die Angelegenheiten wahr, die in der ganzen Stadt einheitlich durchgeführt werden müssen.

Nach der Geschäftsverteilung der Senatsverwaltungen gehören zum Geschäftsbereich der Senatsverwaltung für Inneres ${ }^{2}$ Staats-, Verfassungs und staatliches Organisationsrecht: allgemeines Verwaltungsrecht, Verwaltungsverfahren nach der Verwaltungsgerichtsordnung; Staatsangehörigkeits- und Einbürgerungssachen; Personenstandwesen, Datenschutz, Statistik etc. Die Senatsverwaltung für Inneres ist zuständig auch für allgemeine Fragen der Verwaltungsorganisation: Besonders die Senatsverwaltung für Inneres besteht aus den Abteilungen AV (Allgemeine Verwaltung), I (Verfassungs- und Verwaltungsrecht, Wahlen, Datenschutz, Staatsangehörigkeits-, Namens- und Personenstandwesen, Geschäftsverfahren, Entschädigung und Betreuung der Opfer der nationalsozialistischen Verfolgung), II (Recht des offentlichen Dienstes), III (Öffentliche Sicherheit und Ordnung), V (Organisation und Rationalisierung, Stellenplan, Verwaltungsautomation, Bürotechnik, Innere Dienste). Es gibt auch die Abteilung IV, Landesamt für Verfassungsschutz, obwohl in dem Aufbauorganigram nicht steht, aus dem Grund des Geheimnisses wird nicht bekannt gegeben.

\footnotetext{
*Forschungsassistentin an der Fakultät für politische Wissenschaften, Universität Ankara.

${ }^{1}$ Gliederung der Berliner Verwaltung, Stand: 1. Oktober 1991.

2 Dienstblatl des Senats von Berlin, Teil I, Nr. 2 (17. Februar 1992), s. 13-14.
} 
An der Abteilung V gibt es vier Referade unter der Leitung des Abteilungsleiters. Referat VA ist zuständig für Dienstgebaudeangelegenheiten, Verbesserungsvorshlagwesen: Fachaufsicht über der Fuhrpark und der Verwaltungsdrückerei; Nettobere:hnung von Personal and Versorgungsbezügen.

Referat VC ist zustăndig für Verwaltungsreform; Verwaltungsstrukturkritik; Querschnittsaufgaben der Verwaltungsorganisation in der Hauptverwaltung und den Bezirksverwaltungen; ressortsübergreifendes IT Management.

Die Verwaltung des Landes Berlin ist ein sehr spannendes Thema. Es gibt Zweistufigkeit in der Verwaltung, nămlich die Hauptverwaltung sogenannte Senatsverwaltungen und die Bezirke. Die Bezirke reprăsentieren in Berlin die kommunale Selbstverwaltung. Natürlich kann man die Bezirke nicht so einfach als kommunale Selbsverwaltung wie Gemeinden und Gemeindeverbände in den Flăchenstaaten nennen. Die Begrilndungen werden Schritt für Schritt in den nächsten Teilen geschildert.

Die Problematik dieses Aufsatzes ist als die Verwaltungsreform der Bezirksverwaltungen ausgewăhlt. Aber die bezirksbezogenen Reformmaßnahmen und die Verwaltungskritik sind nur ein Teil der ganzen Reformarbeit der Senatsverwaltung für Inneres, Abteilung VC. Die Reformmaßnahmen sind auch nicht nur mit den hier erwăhnten Diskussionen beschrănkt. Es gibt die Reformvorschlăge über z.B. Personalwesen und die Senatsverwaltungen. Andererseits ist der wichtigste Punk: die Verwaltungsreform wird als kıin einmaliger. Akt, sondern ein stăndiger ProzeB beschrieben.

Dieser Aufsatz besteht neben der Einführung aus vier Teilen. Nach der kurzen Einfuhrung wird in dem zweiten Teil die allgemeine Organisation der Berliner Verwalung erörtet. In dem dritten Teil handelt es sich um die Begründungen der Verwaltungsreform in Berlin. In dem vierten Teil wird über die ausgewăhlten Reformmaßnahmen informiert. Der letzte und vielleicht der wichtigste Teil ist das Schlußwort. Obwohl bisher meistens um deskritive Bemerkungen geht, wurden in diesem letzten Teil auch kritische Analysen vorgenommen. Dieser Kritik über die Berliner Vérwaltung, besonders über die Bezirksverwaltungen, wurde ein Vergleich mit der kommunalen Selbstverwaltung in den Großstădten in der Türkei, weil es in cler Türkei auch zweistufige Verwaltung in den Großstădten wie Istanbul, lzmir und Ankara gibt. Aber man muß niemals vergessen, daß Berlin ein Stadtstaat in der föderativ organisierten Bundesrepublik Deutschland und die Türkei ein einheitlicher zentraler Staat ist. Beim Vergleich soll dieser sehr wichtige Underschied bemerkt werden.

\section{EIN ÜBERBLICK DES AUFBAUS DER BERLINER VERWALTUNG}

Berlin ist ein Stadtstaat genauso wie die Lănder Hamburg und Bremen. Die staatliche und gemeindliche Tătigkeit ist in Berlin nicht getrennt. In der Verfassung von Berlin Artikel 1 Absatz 1 steht: "Berlin ist ein deutsches Land und zugleich eine Stadt". Das bedeutet: die staatlichen und die kommunalen Aufgaben werden von einer einheitlichen Gebietskorperschaften wahrgenommen. 
In Berlin gibt es grundsătzlich zwei verschiedenen Landesverwaltungen:

1. Die unmittelbare Berliner Landesverwaltung ist in Hauptverwaltung und Bezirksverwaltungen gegliedert. Im Sinne des Allgemeinen Zustündigkeitsgesetz ist nur diese unmittelbare Landesverwaltung die Berliner Verwaltung.

Zur Hauptverwaltung gehorren die Mietglieder des Senats (Senatsverwaltungen, z.B. Senatsverwaltung für Inneres, Senatsverwaltung für Soziales, Senatsverwaltung für Justiz etc.), die ihnen nachgeordneten Behörden (z.B. Polizeipräsident, Landeseinwohneramt, Statistisches Landesamt etc.) und nichtrechtsfahigen Anstalten wie die Eigenbetriebe (z.B. BVG, Berliner Verkehrsgesellschaft) ${ }^{3}$.

Nach der Verfassung von Berlin (vom 1. September 1950) war Berlin in 20 Bezirke eingeteilt. Aber diese Zahl ist nach der Wiedervereinigung auf 23 erhơht $^{4}$.

Organe der Bezirksverwaltung sind die Bezirksverordnetenversammlung und Bezirksamt. Zu den Bezirkverwaltung gehören auch nichtrechtsfăhige Anstalten, nämlich Schulen und Krankenhausbetriebe.

2. Die mittelbare- nachgeordnete Berliner Landesverwaltung besteht aus Körperschaften, Anstalten und Stiftungen z.B. berufsstăndische Kammer, Instituten etc. Diese Verwaltung ist in Form von selbststăndigen juristischen Personen des offentlichen Rechts aufgebaut.

Nach der Verfassung von Berlin und dem Allgemeinen Zustăndigkeitsgesetz (AZG) nehmen die Bezirke zwei verschiedene Aufgaben wahr. Eine Gruppe der Aufgaben sind sogenannte bezirkseigene Angelegenheiten. Das bedeutet: Die Bezirke sind zuständig für die Verwaltungsaufgaben, die in den gesetzlichen Regelungen ausdrücklich den Senatsverwaltungen und den ihnen unmittelbar nachgeordneten Behorden und Einrichtungen nicht zugewiesen sind. z.B. die Einzelpersonalangelegenheiten, der großte Teil der sozialen Leistungen etc.

Die andere Gruppe der Aufgaben sind unter der Fachaufsicht einer Senatsverwaltung übertragen. z.B. Ordnungsaufgaben wie die Bau- und Wohnungsaufsicht, die Gesundheits-, Veterinär- und Lebensmittelaufsicht ${ }^{5}$.

\section{DIE BEGRÜNDUNGEN DER VERWALTUNGSREFORM}

Man kann diese Begründungen in zwei Gruppen gliedem:

1. Die allgemeine Begründungen: Die Verwaltungsreformarbeit hat im Jahr 1982 begonnen. Damaliges Abgeordnetenhaus von Berlin hat entschieden, eine Enquete Kommission zu bilden, um über die Verwaltungsreform zu bearbeiten. Der

\footnotetext{
${ }^{3} \mathrm{Vgl}$. Art. 44 Verfassung von Berlin und Art. 2 Abs. 2 Allgemeine Zustänigkeitsgezets.

${ }^{4} \mathrm{Vgl}$. Art 4 Abs. 1 Verfassung von Berlin vom 1. September 1950 zuletz geändert durch Gesetz vom 3. Juni 1991.

${ }^{5} V_{g l}$. Art. 51 Abs. 1-2-3 Verfassung von Berlin.
} 
Zweck war, eine effektive, sparsime und bürgernăhe Verwaltung zu schaffen. Um diesen Zweck realisieren zu können, hatten sie folgende Ziele:

- Gewăhrleistung der Bürgerbeteiligung,

- Vermeiduing von Doppelarbeit.

- Dezentralisierung der Verwaltung.

Nach den Bericht dieser Enquete Kommission wurden folgende Punkte vorgesehen 6 :

- Stärkung der Eigenverantwortung der bezirklichen Verwaltung,

- Änderung der Zuständigkeits -Aufgabenverteilung zwischen Haupt- und Bezirksverwaltung hinsichtlich einer Neuordnung der Vorbehalts-und bezirkseigenen Aufgaben und der Ordnungsaufgaben,

- Änderung der Bezirksverwaltungsorganisation hinsichtlich der Große und Struktur der Bezirksămter,

- Abbau der Bürokratisierung,

- Überleitung von Verwaltungsaufgaben in besser geeignete Organisationsformen oder auf andere Trăger.

Es gibt zwei verschiedene Kritiksgruppen über die Verwalung von Berlin?:

\subsection{Verwaltungskritik:}

- Es gibt eine Monopolstellung in der offentlichen Verwaltung,

- Selbst in den Handlungsbereichen gibt es in Verwaltungen keinen wirksamen Erfolgsmaßstab und damit keine Erfolgshaftung,

- Führung in der Verwaltung ist keinerlei konkreten Optimierzungszwănge unterworfen, ist begrenzt.

- Wenig Innovation und Rationalisierung; Handlungs- und Anpassungsfahigkeit

\subsection{Kritik der Bürger:}

- Das Verwaltungshandeln erscheint undurchsichtig; Zustăndigkeiten sind unklar und überschneiden sich,

- Die Beteiligung der Bürger ist zu wenig oder nur formalistisch,

- Die Verwalung enscheint unbeweglich und unwirtschaftlich.

In dem Bericht über Umsetzung der Arbeitsergebnisse der Enquete Kommission zur Verwaltungsreform wurden folgende Ziele weiterbestimmt:

- Subsidiarităt der Verwaltung. Das heißt: Die Verwaltung soll nur dort tătig sein, wo einzelne Bürger oder Grupprn nicht selbst sinnvoll etwas regeln konnen oder wo ein Handeln der Verwaltung aus manchen Gründen notwendig ist; Prinzip der Subsidiaritat zwischen Staat und Gesellschaft.

${ }^{6}$ ggl. 2. Bericht der Enquete Kommission zur Verwaltungsreform vom 30. Mai 1984: S. 35.

7 Vgl. 2. Bericht, 1984: S. 5. 
- Nach dem Prinzip der innerbehơrdlichen Subsidiarităt sollte der Bürger gemåß Moglichkeit ortsnah und verkehrgünstig alle für ihn wichtigen Verwaltungsstellen finden.

2. Wegen der Situation der Bezirksverwaltung: In Berlin gibt es neben den allgemeinen Problemen wie alle Verwaltungen zusătzlich besondere Probleme wegen der Zweistufigkeit der Verwaltung. Berlin ist ein groBes Gebiet und jetzt die Hauptstadt der Bundesrepublik Deutschland. Deshalb muB Berlin politisch und verwaltungsmaBig untergliedern werden. Diese Zweistufigkeit der Berliner Verwaltung und in dem Verordnung zur Durchführung des Allgemeinen Zuständigkeitsgesetzes (DVO-AZG) ${ }^{8}$. Dieses System ist sowie so effektiv, sofern die Hauptverwaltung und Bezirksverwaltungen in arbeitsteiliger Weise seine Aufgaben erledigt. Aber nur Effektivităt ist nicht reicht. Gleichzeitig muB die Verwaltung wirtschaftlich und bürgernăhe sein. Deswegen soll man die Eigenverantwortung der Bezirksverwaltung vergroßern. Dafür ist das Hauptziel Starkung der eigenverantwortlichen bezirklichen Selbstverwaltung durch Änderung der Zuständigkeits- und Aufgabenverteilung, weil Partner der Bürger soll nur eine Verwaltung sein und möglichst eine regional zustăndige, kompenente und entscheidungsfăhige Verwaltung.

Andererseits haben die Bezirke in Berlin manche Besonderheiten. Diese Besonderheiten in der Organisation machen die Bezirke unterschiedlich von den Gemeinden und Gemeindeverbănden in Flächenstaaten.

\section{Diese Unterschiede sind folgende?:}

2.1. Die Bezirke sind nicht in Form von den Korperschaften oder sonstigen juristischen Personen des offentlichen Rechts. Es kann sich um die rechtliche Konflikte sowohl zwischen Bezirk und Bürger als auch zwischen Bezirksverwaltung und Hauptverwaltung handeln. Wenn ein Bürger eine Klage wegen der Entscheidung des Bezirksamtes vor dem Verwaltungsgericht erhebt, erscheint in diesem Fall als Beklagter das Land Berlin, also nicht das Bezirksamt. Andererseits kann es auch einen Rechtsstreit zwischen den Organen der Bezirksverwaltung und dem Senat oder einer Behorde der Hauptverwaltung geben. Die Körperschaft ist als das Land Berlin dieselbe. Dieser ProzeB soll deswegen als Organstreitverfahren bezeichnen.

2.2. Die Organe der Bezirksverwaltungen haben keine legislativen Befugnisse. Die Berliner Bezirke sind mit der Exekutive beschränkt. Das bedeutet: Die Bezirke dürfen keinen Rechtsnormen erlassen wie die Gemeinden und andere Korperschaften des offentlichen Rechts. z.B. die wichtigste Gemeindesatzung ist für die Bürger die Bebauungspläne.

Dagegen haben Bezirksverordnetenversammlungen in einigen Fallen ein Mitwirkungsrecht bei dem Erlaß von Rechtsverordnungen. z.B. bei der Änderung der Bezirksgrenze und bei der Aufstellung der Bebauungsplane.

2.3. Vielleicht ist die wichtigste Besonderheit der Bezirksverwaltung, daB die Bezirke grundsatzlich keine Etat- und Finanzhoheit haben. Die Haushaltsplane der Bezirke

${ }^{8} \mathrm{Vgl}$. Bericht vom 18.10.1988.

${ }^{9} V_{g l}$. Aufbau und Aufgaben der Berliner Bezirksverwaltung, 1992: 15-16. 
sind Teil des Berliner Hasthalısplans. Eigentlich ist das eine Konsequenz davon, daß die Bezirke keine juristische Körperschaften sind.

2.4. Gegenüber den schon erwăhnten Besonderheiten haben die Bezirke Personalhoheit. Sie sind ganz selbständig für die Einstellung, Entlaßung und Umsetzung der personal innerhalb der Bezirksverwaltung.

\section{DIE REFORMMABNAHMEN}

In den letzten Jahren gibt es eine neue zwar sehr starke Tendenz: Verwaltungsleistungen sollten meßbar und bewertbar sein ${ }^{10}$. Dafür braucht man manche Erfolgskriterien wie Effektivität, Effizienz, Qualităt, Sparsamkeit und Wirtschaftlichkeit staatlicher Leistungen ${ }^{11}$. Die Verwaltungsreform ist deswegen unvermeidbar auch für die Berliner Verwaltung, weil kommunale Reformen sind ein Teil von der Entwicklung im Gesamtverwaltungssystem. In der jüngsten Zeit haben folgende Reformthemen besonders wichtige Rolle gespielt ${ }^{12}$ :

- Rückbau des Staates, Verwaltung,

- Fortsetzung von Demokratisierungsanstrebungen und Schaffung bürgemăherer

- Entbürokratisierung, Rationalisierung,

- Reorganisation des öffentlichen Sektors wie Dezentralisierung und

- Verbessurung des Finanswesen,

- Leistungsorienticrung der offentichen Personalpolitik.

Die Reform der Berliner Verwaltung wird als ein sehr wichtiger Politikschwerpunkt von dem Senat angesehen. Denn die Aufgaben Berlins verlangen dringend eine Verwaltungsreform. Berlin hat jetzt neue Bedürfnisse als Hauptstadt und europäischer Metropole. Wegen dieser neuen Missionen soll man eine Reform unter oben geschilderten Kriterien umsetzen, die an eine leistungsfahige, kostengünstige, demokratische und bürgernähe Verwaltung verbindet ist. Aber inzwischen bleibt zweistufiger Verwaltungsaufbau Berlin gleich.

In dem Bericht, der im Jahr 1993 von der Senatsverwaltung für Inneres Abteilung VC ausgearbeitet ist, stehen die Vorschlăge über die Verwaltungsreform in Berlin. Die ausgewählte vorgeschlagene Reforrnmaßnahmen:

1. Die Aufgabenverteilung zwischen der Hauptverwaltung und den Bezirken soll wieder umsetzen, um die Bezirke zu verstärken und die Bürger- und Ortsnähe der

${ }^{10}$ Helmut Klages, Oliver Haubner, "Qualitïts-und Ërfolgsmerkmale von Organisationen im öfentlichen Bereich," Verwaltung Organisation Personal, Nr. 4/1990, s. 24.

11 Hermann Hill, "Strategische Erfolgsfaktoren in der öfflichen Verwaltung," Schriftenreihe der Hochschule Speyer, Band 112, Duncker und Humblot, Berlin, 1993, s. 29.

${ }^{12}$ Christoph Reichard, "Intemationale Managementtrends," Gerhard Banner und Christoph Reichard (Hrsg.), Kommunale Managementkonzepte in Europa, Köln, Deutscher Gemeindeverlag, 1993, S. 4. 
offentlichen Verwaltung zu erhőhen. Im Rahmen dieser Aufgabenverteilung beschränkt sich die Zustăndigkeit der Hauptverwaltung grundsäłzlich auf ministerielle, gesamt- und hauptstădtische nămlich bezirksübergreifende Angelegenheiten. Die Hauptverwaltung garantiert die Einheitlichkeit der Berliner Verwaltung und die Angleichung der Lebensverhältnisse in der gesamten Stadt.

Für das gesamte Bebauungsplanverfahren ist die Bezirke (95 \%) zustăndig. Im Rahmen dieser Reformarbeit fordem die Bezirke auch die Festsetzung der Bebauungsplane in ihre Zustăndig zu verlagem. Aber die Senatsfachverwaltungen lehnen diese Verlagerung aus dem Grund ab, daB die Festsetzung der Bebauungsplane ein sehr wichtig politisches Steuenungsinstrument ist.

2. Bezirksreform. Das Ziel dieser ReformmaBnahme ist, eine gleichgewichtigen Stadtstruktur zu schaffen. Aus den Gründen der Gewahrleistung der leistungsfahigen Organisation und Wirtschaftlichkeit werden 23 Bezirke auf 12 (höchstens 15) verringert. Aber die existierende Bezirke werden unter Wahrung historisch gewachsener Strukturen zusammengelegt.

Inzwischen wird geplant, die Anzahl der Mitglieder des Bezirksamts pro Bezirk auf 5 einschieBlich des Bezirksbürgermeister zu verringem. Aber andererseits wird die Anzahl der Mitglieder der Bezirksverordnetenversammlungen unverăndert bleiben wegen des ehrenamulichen Engagements und der demokratischen Teilhabe der Bürger.

3. Finanzautonomie der Bezirke. Darüber werden die Vorschläge folgende erwăhnt ${ }^{13}$ :

Zum Zwecke der Stărkung dér politischen und finanziellen Eigenverantwortung der Bezirke wird die Finanzstruktur im Verhăltnis Hauptverwaltung/Bezirke wie folgt neu geordnet: Die Bezirke erhalten zur eigenverantwortlichen Wahmehmung aller ihnen obliegenden Aufgaben Globalsummen mit einem Höchstbetrag für Personalmittel und einem Mindestbetrag für Investitionen zugewiesen, die sie in eigener fachlicher und politischer Verantwortung bewirtschaften...

Den Bezirken wird Teilhabe am Steuer Aufkommen eingeräumt, um Anreize für eine aktive Wirtschaftsförderung-und Ansiedlungspolitik und den Aufbau der dafür erforderlichen Infrastruktur durch die Bezirke zu schaffen. Hierbei ist den besonderen Gegebenheiten der Einheitsgemeinde Rechnung zu tragen, insbesondere sind das Budgetrecht, die verfassungsrechtliche Kontrollfunktion des Abgeordnetenhauses und die Funktion des Rechnunshofes uneingeschränkt zu erwahren...

4. Globalsteuerung. Eine zentrale Globalsteuerung der Verwaltung, wie bei modernen Unternehmen der Wirtschaft wird vorgeschlagen. Durch dieses zentrale Globalsteuerungsmodell wird eine weitgehende Verselbstandigung von

${ }^{13}$ Der Bericht uber Reform der Berliner Verwaltung, 1993: S. 6. 
Verwaltungseinheiten und eine dezentrale ganzheitliche Fach-, Personal-, Organisations-, Ressourcen- und Ergebnisverantwortung gewährleistet. Dieses Modell wird zunächst in den Senatsverwaltungen für Inneres und Soziales sowie in drei Bezirken erprobt werden. Wenn es erfolgreich wird, soll es flăchendeckend in der Berliner Verwaltung eingeführt werden.

5. Als die Reformmaßnahme wird die Übertragung der offentlichen Aufgaben auf freie und private Träger zum Ausdruck gebracht. Das Kriterium ist dafür der Grundsatz der Subsidiarităt und die Wirtschaftlichkeit der offentlichen Aufgabenwahrnehmung. Besonders an wie folgenden Bereichen:

- Wäschereien,

- Gebăudereinigung,

- Krankenpflegedienst,

- Parkhăuser,

- Sportanlagen etc.

Eine solche Übertragung wird die Leistungsfähigkeit der Verwaltung verbessem und gleichzeitig gewährleisten, die Aufgaben konsengünstiger zu erfüllen.

6. Es wird ganz stark betont, daß Berlin als Einheitsgemeinde bleibt. Das heißt: Die staatliche und gemeindliche Tätigkeiten sind nicht getrennt, und die Bezirke bleiben immer noch in gleichem Form, also sie haben Selbstverwaltungseinheiten ohne Rechtspersönlichkeit.

\section{SCHLUBWORT}

1. Die Reformarbeit; die im 1982 begonnen hat, war nicht die erste. Die Aspekte der Verwaltungsreform waren früher ehor Entbürokratisierung. Heute ist der Themenkreis viel eher unter dem Aspekt der Bürgemähe, der Sparsamkeit und Effektivität. In künftigen Zeiten wird man eventuell wiederum ganz andere Aspekte zugrunde legen. Das bedeutet: Die Verwaltungsreform ist ein ständiger Prozeß.

2. Als eine Reformmaßnahme wird die Verringerung der Anzahl der Bezirke auf 12 vorgeschlagen. Gleichzeitig werden auch die Anzahl derMitglieder des Bezirksamts und der Abteilungen des Bezirksamts verringert. In diesem Punkt soll man folgende Fragen zur Diskussion stellen:

- Die Verringerung der Anzahl der Bezirke (fast auf die Halfte) ist unrealistisch. Im Sinne der Globalsteuerung ist diese Verringerung konsequent. Aber klar ist: Je größer eine Organisation ist, desto geringer ist Partizipationschance der Bürger an die Verwaltung.

- Wenn die Anzahl der Bezirke verringert wird, wird die Verantwortung der jeweiligen Bezirke unvermeidbar erhöht. Dennoch wird die Anzahi der Abteilungen sowie der Mitglieder des Bezirksamts verringert. Es sieht so aus, als ob es zwischen dem Ziel und dem Mittel eine Paradoxie gäbe.

- Es geht um eine juristische Diskussion über die Veränderung der Bezirksanzahl in Berlin. Es ist noch zweifelhaft, ob es sich um ein verfassungsänderndes Gesetz handeln 
muß oder ob ein einfaches Gesetz ausreicht, weil nach Artikel 4 Absatz 2 der Verfassung von Berlin die Zahl und die Grenzen der Bezirke nur durch Gesetz geăndent werden kornnen.

3. Die Bezirke reprăsentieren in Berlin bürgernăhe und demokratische Selbstverwaltung ${ }^{14}$. Sie haben wesentliche ortliche Aufgaben. Aber die Organe der Bezirke besizen keine legislativen Befugnisse. Die Bezirke düren keine Rechtsnormen erlassen. Sie haben nur in einigen Fallen ein Mitwirkungsrecht. Andererseits gibt es viele Vorteile, eine juristische Person des bffentlichen Rechts zu sein. Als Beispiel: Man darf einen eigenen Haushalt haben oder bei Gericht darf man Klager sein. Wegen der Einheit des Landes Berlin und der Stadt Berlin scheint so, als ob die Bezirke ein Teil der Hauptverwaltung wăren.

4. Das System der GroBstadtverwaltung in der Türkei besteht aus zwei verschiedenen Ebenen, die Zweistufige Verwaltung, wie in Berlin. Oben gibt es eine Hauptverwaltung, die einen Großstadtmagistrat, einen Oberbürgermeister und einen GroßstadtausschuB hat. Diese Hauptverwaltung ist auch eine kommunale Selbstverwaltung. Unten gibt es die Bezirksverwaltungen. Jeder Bezirk hat seinen eigenen Bürgermeister, Magistrat und BezirksausschuB. Die ortlichen Aufgaben sind zwischen der Hauptverwaltung und den Bezirken aufgeteilt wie in Berlin. Nach dem veriikalen Finanzausgleichsprinzip werden die Einnahmen zwischen der Zentralverwaltung, der Hauptverwaltung und den Bezirken aufgeteilt.

Die Bezirke sind juristische Personen des offentlichen Rechts. Sie haben jeweils ihren eigenen Haushalt, sind aber trotzdem hinsichtlich der Einnahmen abhängig von der Zentralverwaltung und der Hauptverwaltung.

Das zentralistische Verwaltungssystem, das in der Türkei angewendet wird, ist vom französischen Verwaltungssystem des 19. Jahrhundert übernommen worden. Deshalb läı sich dieses System nicht an die gegenwărtigen Bedingungen anpassen. Besonders seit 1980 gibt es deswegen Reformbestrebungen auch auf dem Gebiet der kommunalen Selbstverwaltung in der Türkei. Die Reduzierung der Anzahl der kommunalen Selbstverwaltungen bildet einen wichtigen Reformvorschlag, weil viele kommunale Selbstverwaltungen ineffizient arbeiten. Die Venteilung der Aufgaben und Einnahmen ist nicht richtig geregelt. Deshalb gibt es manchmal fehlende Aufgaben oder manche Aufgaben werden von mehr als einer Verwaltung erfüllt. Es ist eine Tatsache, daB sowohl verikale Fehlkoordination als auch horizontale existiert.

5. Die Hauptverwaltung hat in Berlin die Aufsichtsbefugnisse über den Bezirken. Die Aufsichtsmittel ${ }^{15}$ sind Informationsrecht (die Aufsichtsbehơrden können Auskünfte oder Berichte von den Bezirken zur Erfüllung ihrer Aufgaben fordem), Aufhebungsrecht (der Senat kann Beschlüsse und Anordnungen bezirklicher Organe, die das bestehende Recht verletzen, aufheben) und Anweisungsrecht (wenn es erforderlich ist, kann der Senat dem zustăndigen Organ Weisungen geben, damit es innerhalb bestimmter Fristen erforderliche Beschlüsse faß $\mathrm{A}$ ). Aber hier gibt es einen sehr wihctigen Punkt: Die Hauptverwaltung macht von Aufsichtsbefugnissen nur im Rahmen der Rechtsvorschriften Gebrauch. Das bedeutet: Es handelt sich um keinen Wilkürakt. Andererseits ist das auch

${ }^{14} V_{\text {gl. Art. } 50}$ Abs. 2 Verfassung von Berlin.

$15 \mathrm{~V}_{\mathrm{g}}$. Aubau und Aufgaben der Berliner Bezirksverwaltung, 1992: S. 23-24. 
wichtig, daß die bezirkseigenen Aufgaben und die übertragenen Aufgaben hinsichtlich der Aufsicht von der Hauptverwaltung differenziert sind. Die Hauptverwaltung darf die bezirkseigenen Aufgaben nur hinsichtlich der Rechtmaßigkeit kontrollieren, weil diese Aufgaben lediglich ortliche Anegelegeṇheiten sind. Aber die Hauptverwaltung darf die übertragenen Vorbehaltsaufgaben hinsichtlich der Zweckmåßigkeit auch kontrollieren, weil sie einer einheitlichen Durchführung bedürfen.

Wenn in diesem Punkt ein Vergleich mit der Türkei gemacht werden soll, kann man folgendes feststellen: Die Aufsichl der Hauptverwaltung und auch gegebenenfalls der Zentralverwaltung ist eher eine solche der ZweckmäBigkeit. Aber von diesen Fachaufsichtsbefugnissen werden meistens von den zustandigen Behorden willkürlich Gebraucht gemacht. Entweder aufgrunid der Lücken der Rechtsvorschriften, oder der nicht klar formilierten Rechtsvorschriften bzw. des Mißtrauens der Zentralverwaltung entstehen derartige Willkürakte. Ein sehr wichtiges Beispiel dazu von der tïrkischen Verfassung ${ }^{16}$ : Die Fachaufsicht der Zentralverwaltung wurde in der Verfassung klar herausgestellt. Die Zentralverwaltung hat die Fachaufsichtsbefugnisse uber die kommunalen Selbstverwaltungen, um

- die Erfüllung der ơrtichen Aúgabeñ zu gewăhrleisten,

- die Einheit des ơffentlichen Dienstes zu gewăhrleisten,

- den Schutz des offentlichen Interesses und die vollstăndige Deckung der offentlichen Bedürfnisse zu gewathrleisten.

6. Es gibt natürlich viels: Schwierigkeiten, um eine grundlegende Verwaltungsreform durchzusetzen. Mangeln des Durchsetzungsverm §gen, aber vor allem auch Wiederstand von Abgeordnetenlaus und Senat auf eine harte Probe stellen. Aber andererseits die längerfrgistigen Entwi:klungen von Wirtschaft und Verwaltung in Berlin ließen keine andere Wahl.

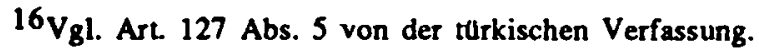

